\title{
Clarithromycin Stops Lung Function Decline in Airway-Centered Interstitial Fibrosis
}

\author{
S. Jouneau ${ }^{\mathrm{a}} \quad$ M. Kerjouan ${ }^{\mathrm{a}} \quad$ S. Caulet-Maugendre ${ }^{\mathrm{b}} \quad$ S. Guillot ${ }^{\mathrm{c}} \quad$ C. Meunier ${ }^{\mathrm{d}}$ \\ B. Desrues ${ }^{a} \quad$ P. Delaval ${ }^{a}$
}

a Service de pneumologie, b Service d'anatomie pathologique et de cytologie, 'Service des explorations

fonctionnelles respiratoires, et ${ }^{d}$ Service de radiologie, CHU de Rennes, Université de Rennes 1, Rennes, France

\section{Established Facts}

- Pulmonary fibroses are progressive and fatal lung diseases lacking effective treatment.

- Airway-centered interstitial fibrosis (ACIF) seems to be cortico-resistant, with inevitable loss of lung function and a high mortality rate in the absence of lung transplantation.

\section{Novel Insights}

- This is the first report of a patient diagnosed with ACIF and stabilized with clarithromycin after the failure of systemic corticosteroids.

- There is a need to confirm this benefit in other patients to ascertain the anti-inflammatory effect of macrolides in ACIF.

\section{Key Words}

Pulmonary fibrosis - Macrolides · Anti-inflammatory effect $\cdot$ Interstitial lung disease $\cdot$ Lung function decline

\section{Abstract \\ Airway-centered interstitial fibrosis (ACIF) is a distinct type of lung interstitial fibrosis characterized by lesions centered on the airways. Several cases reported in the literature showed little to no effect of corticosteroids and a high mor-}

tality rate in the absence of lung transplantation. No other efficient approach is described for the treatment of this type of fibrosis. We report for the first time the case of a 44-yearold patient diagnosed with ACIF on surgical lung biopsy and stabilized with clarithromycin after failure of systemic corticosteroids. We need to confirm this benefit in other patients to ascertain the anti-inflammatory effect of macrolides, which are far less harmful compared to corticosteroids or immunosuppressant drugs.

Copyright $\odot 2012$ S. Karger AG, Basel

\section{KARGER}

Fax +41613061234

E-Mail karger@karger.ch

www.karger.com
(C) 2012 S. Karger AG, Basel

0025-7931/13/0852-0156\$38.00/0

Accessible online at:

www.karger.com/res
Dr. Stephane Jouneau

Respiratory Department, Pontchaillou Hospital, Rennes 1 University

2, rue Henri-Le-Guilloux

FR-35033 Rennes (France)

E-Mail Stephane.jouneau@chu-rennes.fr 


\section{Introduction}

Airway-centered interstitial fibrosis (ACIF) is a distinct type of lung interstitial fibrosis characterized by lesions centered on the airways. Churg et al. [1] described 12 cases in 2004; these reports were followed by case studies from other authors [2-8]. Corticosteroids seem to have little to no effect, and the mortality rate is high in the absence of lung transplantation $[1,5,7]$.

We report herein the first case of a patient diagnosed with ACIF stabilized with clarithromycin after the failure of systemic corticosteroids.

\section{Case Report}

A 44-year-old Caucasian patient with a smoking history of 10 pack-years who had stopped smoking 20 years prior and without a remarkable medical history or long-term medication was referred to our center because of progressive dyspnea and chronic bronchitis. He had bilateral crackles in both lower lung zones. No digital clubbing or extrathoracic finding was present. High-resolution chest CT scan showed predominant subpleural intralobular reticulations in both lung bases, associated with traction bronchiectasis and scissural distortion (fig. 1). Ground glass opacities were also found without honeycombing. Arterial blood gas analysis at rest in room air was normal. Pulmonary function tests (fig. 2) revealed a pure restrictive pattern with a TLC at $47 \%$ and FVC at $55 \%$ of the predicted values. The DLCO was decreased at $43 \%$ of the predicted values. The patient walked $560 \mathrm{~m}$ in $6 \mathrm{~min}$ in room air with a decrease in pulsed oximetry from $98 \%$ at rest to $86 \%$ after $6 \mathrm{~min}$. No systemic inflammation (CRP $=2.3 \mathrm{mg} / \mathrm{l})$, liver, renal or heart dysfunction was observed. Auto-immunity (anti-nuclear antibodies, ANCA, rheumatoid factors), HIV serology, and bird breeders' and farmers' lung serologies were negative. The bronchoalveolar lavage demonstrated 336,000 cells $/ \mathrm{ml}$ with 75\% macrophages, 9\% neutrophils, 9\% eosinophils, and 7\% lymphocytes (41\% CD4+/39\% CD8+).

A surgical lung biopsy was performed (fig. 3) and showed interstitial fibrosis centered on membranous and respiratory bronchioles. Muscle hyperplasia was observed in the bronchiolar wall. No honeycombing or granulomatous lesion was observed; additionally, no polypoid plug was observed within the distal bronchioles. This disorder was very similar to the entity describe by Churg et al. [1]; therefore, we considered ACIF as the final diagnosis.

The patient was treated with $1 \mathrm{mg} / \mathrm{kg}$ of oral prednisolone for 3 months in association with esomeprazole $40 \mathrm{mg}$ /day without any efficacy. Prednisolone was then tapered progressively to $40 \mathrm{mg} /$ day, and esomeprazole was continued at the same dose. The pulmonary function tests worsened during these treatments (fig. 2). Therefore, 6 months later, clarithromycin $(500 \mathrm{mg} /$ day for 1 month and then $250 \mathrm{mg} /$ day) was initiated with a tapered dose of corticosteroids until reaching $20 \mathrm{mg} /$ day as a maintenance treatment. The lung volumes improved slightly and then stabilized at 47,44 , and $47 \%$ for TLC, FVC, and $\mathrm{FEV}_{1}$, respectively, after 20 months of follow-up (fig. 2). No change in chest CT scan, arterial blood gases, DLCO, or a walking distance of 6 min was reported.

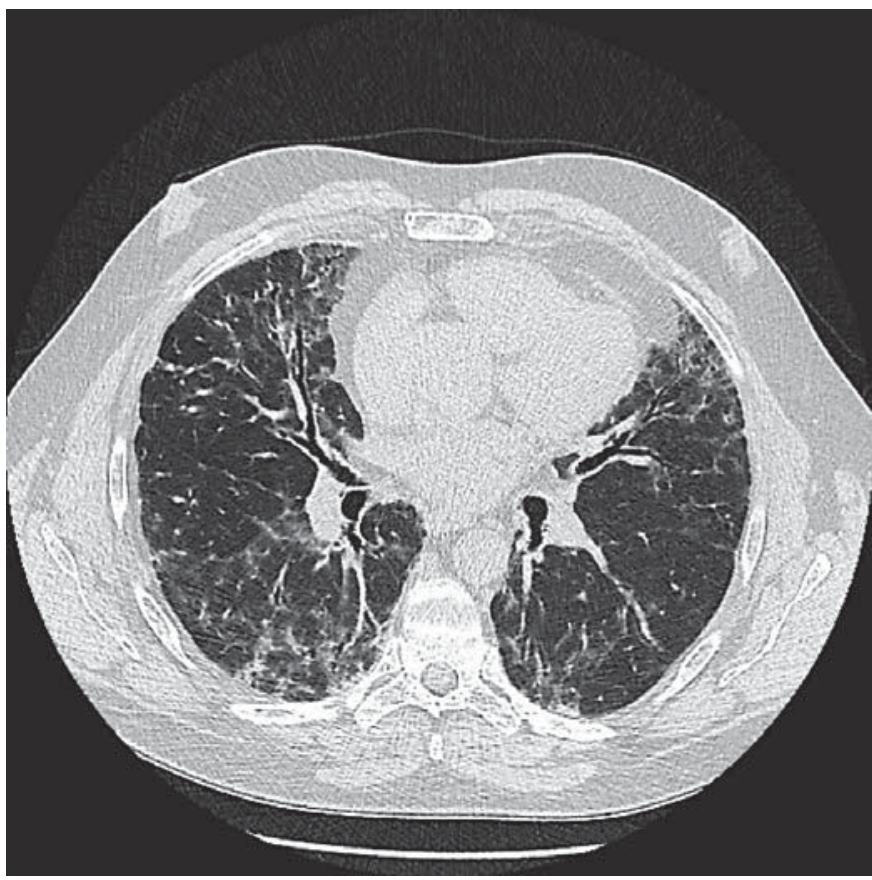

Fig. 1. High-resolution chest CT scan demonstrating the interstitial lung disease with subpleural intralobular reticulations predominant in both lung bases, associated with traction bronchiectases and scissural distortion.

\section{Discussion}

This is the first description of a beneficial response to clarithromycin in a patient with ACIF.

As environmental or occupational exposure is considered to be a strong risk factor for ACIF [1-4, 6-8], we carefully reviewed any exposure in our patient. We found only a mild smoking history (10 pack-years, cessation of smoking for 20 years). No animal contact, including contact with birds, was demonstrated.

Histology is the only method of diagnosis because the HRCT is nonspecific. Peribronchiolar reticulations and ground glass opacities associated with bronchiolectasis on chest HRCT correspond to the pathological presentation with periairway fibrosis. As described by Colombat et al. [6], this airway centricity and the variable importance of the accompanying alveolar interstitial fibrosis indicate that the fibrosis process probably originates in the periairway tissue.

Churg et al. [1] treated the patients with prednisone (0.5-1 mg/kg/day) for 1-3 months followed by inhaled corticosteroids and inhaled bronchodilators as a long-term treatment or followed by progressive reduction toward a 
Fig. 2. Graph showing the evolution of lung volumes with respect to medications. The lung volumes decreased despite the corticosteroids and were stabilized with the initiation of clarithromycin.

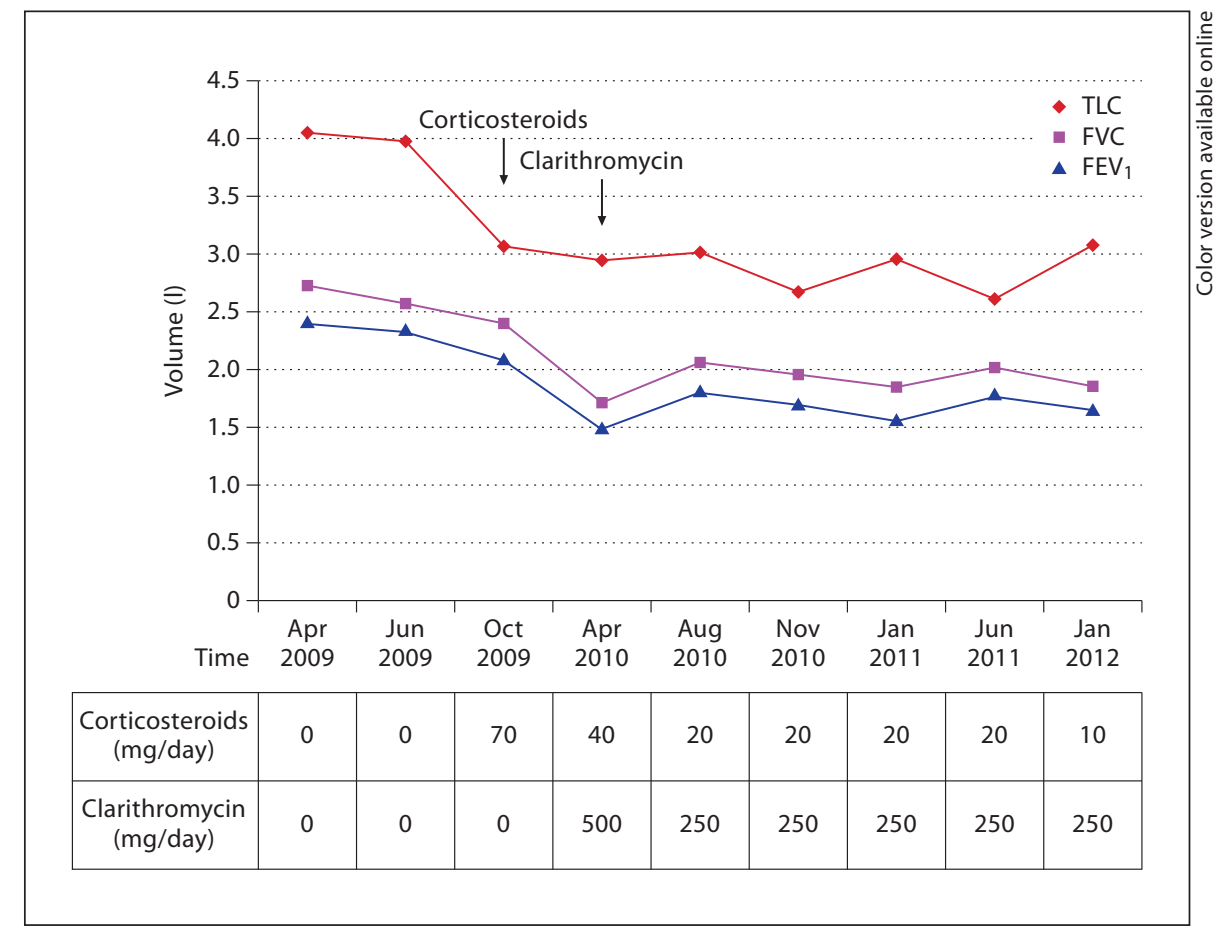

Fig. 3. Surgical lung biopsy with hematineosin-safran staining at magnifications of $\times 20(\mathbf{a})$, and $\times 100(\mathbf{b})$. We observed interstitial fibrosis centered on membranous and respiratory bronchioles associated with smooth muscle hyperplasia. No polypoid bronchiolar plug or constrictive features were present. a The architecture of the lung is relatively preserved, excepted over bronchiolar areas, which are centered by aggregates of lymphocytes (framed). b A dense ring of chronic inflammatory cells surrounds a residual healed bronchiole lined by cuboidal epithelial cells (arrow).
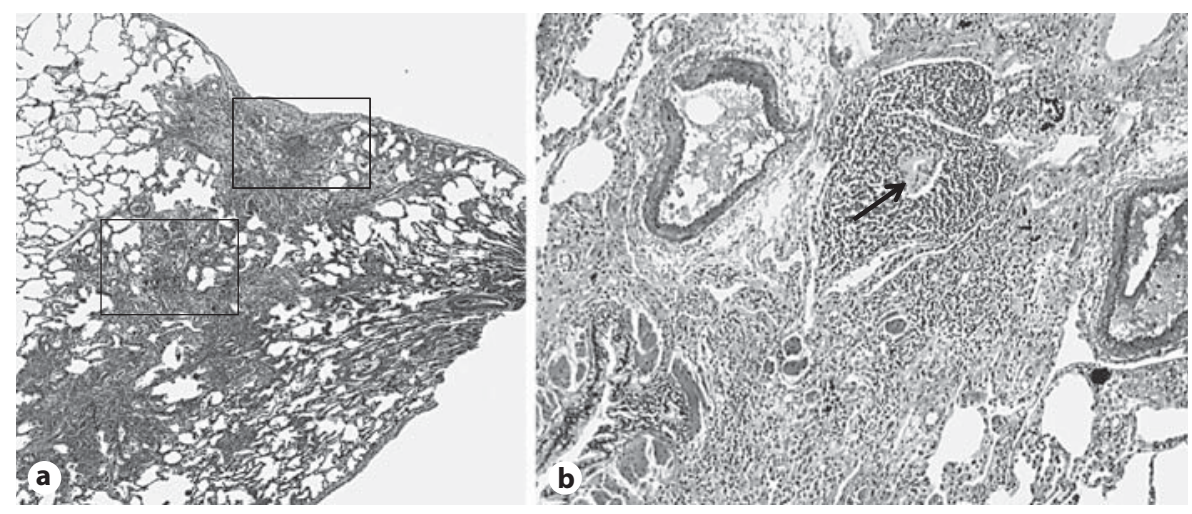

maintenance level of 5-15 mg/day [1]. Only 3 out of 12 patients improved with corticosteroids. The following cases described in the literature demonstrated the inefficacy of corticosteroids in this disease $[5,7,8]$. Azathioprine in addition to corticosteroids was used once without efficacy [8]. In contrast, cyclosporine and corticosteroid treatment demonstrated disease improvement in one case of clear bird-related hypersensitivity pneumonitis; in addition, allergen withdrawal was also performed in this case [4]. The only treatment described as efficient in ACIF was lung transplantation as reported by Colombat et al. [7].
Macrolide antibiotics appear to possess anti-inflammatory properties independent of their antibiotic effect and have been shown to be beneficial in a number of pulmonary disorders, mainly in diffuse panbronchiolitis, and in other chronic pulmonary inflammatory disorders, including cystic fibrosis, non-cystic fibrosis bronchiectases, chronic obstructive pulmonary diseases, and asthma [9]. Although the exact mechanism for the beneficial effect of macrolides has not been completely clarified, it is clearly not related to their antibiotic properties. Macrolides have been described to prevent airway epithelial 
damage and reduce mucus hypersecretion [10]. The immunomodulating effects of macrolides have been demonstrated; in particular, neutrophil accumulation, adhesion and apoptosis are clearly reduced, which results in markedly decreased airway neutrophilia [10]. Effects on cellular immunity with an impact on T-cell regulation and antigen presentation have also been demonstrated [10].

In summary, our case is the first report of ACIF showing a favorable response to treatment with clarithromycin. It will be necessary to confirm this benefit in other patients to ascertain this effect of macrolides, which have fewer side effects compared to immunosuppressant drugs, especially opportunistic infections.

\section{Acknowledgments}

The authors thank Marianne Kambouchner (Hôpital Avicenne, Paris, France) for her technical expertise.

\section{Financial Disclosure and Conflicts of Interest}

The authors have no conflicts of interest.

\section{References}

$>1$ Churg A, Myers J, Suarez T, Gaxiola M, Estrada A, Mejia M, Selman M: Airway-centered interstitial fibrosis: a distinct form of aggressive diffuse lung disease. Am J Surg Pathol 2004;28:62-68.

-2 Gaxiola M, Buendia-Roldan I, Mejia M, Carrillo G, Estrada A, Navarro MC, Rojas-Serrano J, Selman M: Morphologic diversity of chronic pigeon breeder's disease: clinical features and survival. Respir Med 2011;105: 608-614.

$\checkmark 3$ Reghellin D, Poletti V, Tomassett S, Dubini A, Cavazza A, Rossi G, Lestani M, Pedron S, Daniele I, Montagna L, Murer B, Chilos M: Cathepsin-k is a sensitive immunohistochemical marker for detection of microgranulomas in hypersensitivity pneumonitis. Sarcoidosis Vasc Diffuse Lung Dis 2010; 27:57-63.
4 Hara Y, Kobayashi H, Osoreda H, Kanoh S, Motoyoshi K, Ozeki Y: Bird-related chronic hypersensitivity pneumonitis demonstrating the fluctuation of disease activity accompanied with environmental changes. Nihon Kokyuki Gakkai Zasshi 2008;46:1045-1049.

$\checkmark 5$ Yi XH, Chu HQ, Cheng XM, Luo BF, Li HP: Idiopathic airway-centered interstitial fibrosis: report of two cases. Chin Med J (Engl) 2007;120:847-850.

6 Fenton ME, Cockcroft DW, Wright JL, Churg A: Hypersensitivity pneumonitis as a cause of airway-centered interstitial fibrosis. Ann Allergy Asthma Immunol 2007;99: 465-466.
Colombat M, Groussard O, Taille C, Marrash-Chahla $\mathrm{R}$, Brugiere $\mathrm{O}$, Mal $\mathrm{H}$, Leseche G, Fournier M, Degott C: Lung transplantation in a patient with airway-centered fibrosis. Am J Surg Pathol 2004;28:1540-1542.

8 Serrano M, Molina-Molina M, Ramirez J, Sanchez M, Xaubet A: Airway-centered interstitial fibrosis related to exposure to fumes from cleaning products. Arch Bronconeumol 2006;42:557-559.

$\checkmark 9$ Altenburg J, de Graaff CS, van der Werf TS, Boersma WG: Immunomodulatory effects of macrolide antibiotics. 2. Advantages and disadvantages of long-term, low-dose macrolide therapy. Respiration 2011;81:75-87.

-10 Altenburg J, de Graaff CS, van der Werf TS, Boersma WG: Immunomodulatory effects of macrolide antibiotics. 1. Biological mechanisms. Respiration 2011;81:67-74. 